\title{
Glycosaminoglycans: Anticoagulant and Nonanticoagulant Actions: A Short History of Symposia Held at Villa Vigoni*
}

\author{
Job Harenberg, $\mathrm{MD}^{1}$ Benito Casu, $\mathrm{PhD}^{1}$ Roland Krämer, $\mathrm{PhD}^{1}$ Giangiacomo Torri, $\mathrm{PhD}^{1}$ \\ Annamaria Naggi, $\mathrm{PhD}^{1}$ Sandra Krämer, $\mathrm{BCs}^{1}$ \\ ${ }^{1}$ Clinical Pharmacology, Faculty of Medicine Mannheim, Ruprecht- \\ Karls University of Heidelberg, Mannheim, Germany \\ Semin Thromb Hemost 2014;40:831-836.

\begin{abstract}
Address for correspondence Job Harenberg, MD, Clinical Pharmacology, Faculty of Medicine Mannheim, Ruprecht-Karls University of Heidelberg, Theodor Kutzer Ufer 1-3, D-68167 Mannheim, Germany (e-mail: job.harenberg@medma.uni-heidelberg.de).
\end{abstract}

\author{
Abstract \\ Keywords \\ - glycosaminoglycans \\ - heparin \\ - low-molecular-weight \\ heparin \\ - thromboembolism \\ - nonanticoagulant \\ effects
}

Heparin, a sulfated polysaccharide belonging to the family of glycosaminoglycans, was discovered in the beginning of the 20th century and was initially identified as a procoagulant isolated from liver tissue. After the first application in patients approximately 30 years later, further purification identified the major as well as minor, but important, component units of the complex chain mixtures constituting heparin and the multiplex actions became a scientific challenge recently. A series of "Glycosaminoglycan symposium-anticoagulant and nonanticoagulant actions" developed over the past 20 years and focused on this topic has published research data in three issues of Seminars in Thrombosis \& Hemostasis and in several other international scientific journals. The latest developments on the methods of analysis, the synthesis, the degradation by heparanases and the nonanticoagulant effects in tumor growth, in anti-inflammatory diseases, and in Alzheimer diseases as presented in the 21st symposium are summarized in the present overview on the occasion of the 40th anniversary of the journal with special reference to the journal's founding Editor in Chief, Eberhard F. Mammen.

Heparin was first described by Howell and McLean when they analyzed a thromboplastin substance from the brain and later from liver. Jay McLean was aware of several research results from Germany reporting on peptones from organs acting as thromboplastin. ${ }^{1}$ In 1915 and 1916, he purified in the laboratories of W. H. Howell something from the heparphospatide which contaminated the cephalins obtained also from other brain and heart tissues without telling Howell about the results. $^{2}$ When Howell became aware of the data his student had published, he went on to analyze the contaminated cephalin and identified them as anticoagulants naming them antithrombin and heparin. ${ }^{3}$ Two batches of the purified compound were injected intravenously into dogs

\footnotetext{
* Dedicated on the 40th anniversary of Seminars in Thrombosis $\mathcal{E}$ Hemostasis to Eberhard F. Mammen, Founding Editor and Editor-inChief of this journal until 2008.
}

published online November 6, 2014
Issue Theme A Short History of Thrombosis and Hemostasis: Part II (40th Year Celebratory Issue); Guest Editor: Emmanuel J. Favaloro, PhD, FFSC (RCPA).

to demonstrate the anticoagulant heparin inhibition of blood coagulation. $^{4}$

The founding Editor in Chief of Seminars in Thrombosis $\mathcal{E}$ Hemostasis, Eberhard Mammen, contributed to the pathophysiology of blood coagulation and the biological actions of glycosaminoglycans from the mid-20th century. ${ }^{5}$ In 1962, he described the benefit of heparin in many indications such as hemodialysis patients. ${ }^{6}$ During the period of discovery of low-molecular-weight heparin (LMWH), he investigated their pharmacodynamic effects and speculated about their reduced risk of heparin-induced thrombocytopenia. ${ }^{7}$ In a preface to a Glycosaminoglycan Symposium held at Villa Vigoni, he wrote:

The introduction of LMW heparins has opened new avenues for the management of patients with

Copyright $\odot 2014$ by Thieme Medical Publishers, Inc., 333 Seventh Avenue, New York, NY 10001, USA.

Tel: +1(212) 584-4662.
DOI http://dx.doi.org/ 10.1055/s-0034-1395152. ISSN 0094-6176. 
thromboembolic diseases. In contrast to regular heparin, the mean molecular weights are smaller and represent about half of that of regular heparin. Yet, they are still somewhat heterogeneous and seem to differ from preparation to preparation in some of their biologic properties. No two LMW heparins are alike. In spite of these differences, certain commonalities do exist, which may represent an advantage over unfractionated heparin, especially in their use as a prophylactic antithrombotic modality: 1. LMWHs are at least as effective, if not more effective, as antithrombotic agents when compared with unfractionated heparin. 2 . The rate of absorption from the subcutaneous depots is more complete, leading to a longer efficacy in vivo. This translates to a once a day injection instead of the twice a day use of unfractionated heparin. 3. There are suggestions that their use is associated with less bleeding than unfractionated heparin, although the final outcome awaits completion of many ongoing clinical trials. LMW heparins are not free of bleeding complications, but haemorrhages seem to occur that are dose dependent. 4. The overall incidence of heparin-induced thrombocytopenia seems to be markedly reduced with LMW heparins. It is still encountered in an occasional patient and may be related to the preparation used. There is reason to believe that heparin fractions free of heparin-induced thrombocytopenia will be forthcoming. As LMW heparins are used therapeutically, new dosage schemes and new laboratory tests will have to be developed to assure maximum safety for the patients. ${ }^{8}$

In 2002, Eberhard Mammen (-Fig. 1) participated in one of the series of the Glycosaminoglycan Symposia held in the Villa Vigoni at Menaggio situated at Lake Como in north Italy. The participants of the symposia published articles within Seminars in Thrombosis \& Hemostasis subsequent to the 3rd, 10 th, and 14 th symposium, in volumes each appearing 1 year

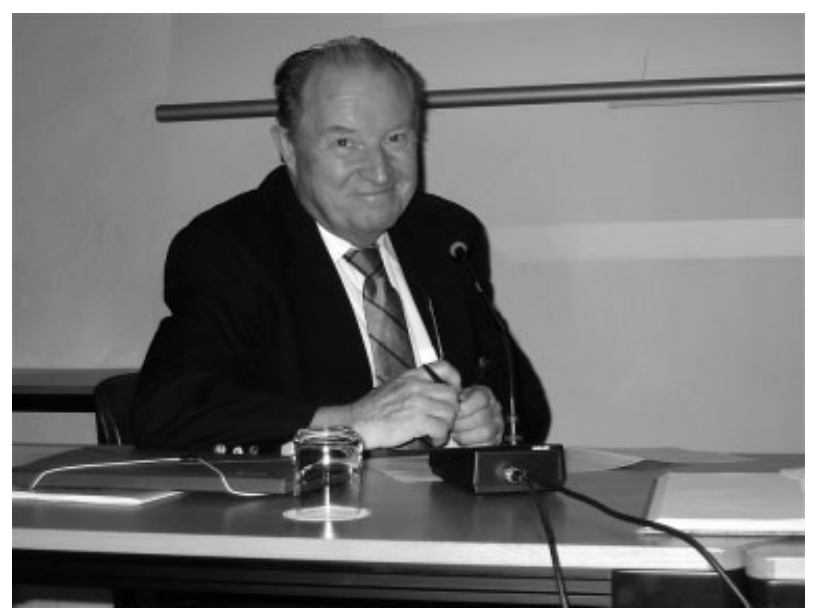

Fig. 1 Eberhard Mammen at the 10th Glycosaminoglycan Symposium, September 20, 2002. later (in $1994,{ }^{9} 2002,,^{10}$ and $2007^{11}$ ). In the preface of the volume of 2002, Mammen wrote that

...most widely known GAGs are heparins and LMWHs and they are best known for their anticoagulant properties. Although the clinical usefulness of heparins cannot be disputed, there are serious side effects associated with their use, more with UFH than with LMWH. These problems have spurred continuous efforts to develop modifications of UFH in order to make compounds safer without compromising the desired anticoagulant activities. ${ }^{8}$

The subsequent symposia continued with the presentation of research on the various problems of anticoagulation and are also presented during the 22 nd edition of the symposium.

\section{Structural Analysis of Glycosaminoglycans}

Antithrombin is required to potentiate the anticoagulant action of heparin LMWH, ${ }^{12}$ fondaparinux, ${ }^{13}$ and other heparin-derived oligosaccharides on coagulation proteases. ${ }^{14}$ Structural aspects of heparins and derivatives are analyzed today using high performance size exclusion chromatography, ${ }^{15}$ capillary electrophoresis, ${ }^{16}$ antithrombin affinity or other matrices in connection with electrophoreses methods, ${ }^{17}$ nuclear magnetic resonance techniques, ${ }^{18}$ matrix-assisted laser desorption/ionization timeof-flight mass spectrometry ${ }^{19}$ and fast atom bombardment, electrospray ionization, and tandem mass spectrometry. ${ }^{20}$ These methods were designed to differentiate isomeric heparin disaccharides, to determine sulfation positions and uronic acid epimerization in oligosaccharides of chondroitin sulfate (another member of the GAG family), and to study the effect of the positions of sulfate groups on heparin binding. ${ }^{20}$

\section{Anticoagulant Actions of Glycosaminoglycans}

The effect of glycosaminoglycans on the blood coagulation system depends on its affinity to bind to antithrombin and results in inhibition of the activity of many serine proteases involved in this process. The initial coagulation cascade described by Ratnoff and Menzie ${ }^{21}$ included an intrinsic and an extrinsic pathway activation of the factor-tenase complex. The steps followed by conversion of prothrombin to thrombin to initiate clot formation by splitting fibrinopeptides A and B from the $\alpha$ and $\beta$ chains of fibrinogen to build fibrin. Substantial modifications of this initial concept adapted the system of blood coagulation to include activation of both pathways through tissue factor (TF), activation of the fibrinolytic system by coagulation proteases factors XII and thrombin and inactivation of fibrinolysis by activating thrombin-activated fibrinolysis inhibitor, multiple functions of thrombin by feedback activation of factor VIII, inhibition of factors V and VIII through binding to endothelially located thrombomodulin via the proteins $C$ and $S$ pathway, activation of factor XIII to activate glutaminase for stabilization of fibrin strains, binding to and activating protein-activating receptors on cell surfaces of platelets and leukocytes. ${ }^{22}$ 


\section{Methods to Determine Anticoagulant Effects of Glycosaminoglycans}

Overwhelming literature is available on the determination of the anticoagulant effects of various glycosaminoglycans, including heparins, LMWH, fondaparinux and heparin-derived oligosaccharides as well as other polysulfated polysaccharides. ${ }^{23}$ Many methods for the determination of heparins were presented at the Glycosaminoglycan symposia and some of them are commercially available today. The prothrombinaseinduced clotting time revealed a higher sensitivity over prothrombin time and activated partial thromboplastin time assays for determination of heparins, LMWH, and fondaparinux. This assay was also independent of anticoagulant effects of a concomitant therapy with vitamin $\mathrm{K}$ antagonists. ${ }^{24} \mathrm{~A}$ specific assay was described for fondaparinux because heparanase does not degrade the pentasaccharide sequence constituting the antithrombin-binding region in contrast to other sequences in heparin and LMWH. ${ }^{25}$ The concentration of heparin can be detected by a polycationic ruthenium compound which quantifies heparin by monitoring $630 \mathrm{~nm}$ fluorescence. The compound is an example of a nonpolymeric low-molecular-weight agent which neutralizes the anticoagulant activity of heparin and LMWH in plasma samples. ${ }^{26}$ The fluorescent perylenediimide probes modified with $2,4,6$, or 8 ammonium groups were synthesized and their binding to the antithrombotic drug heparin was studied by fluorescence spectroscopy in solution. The polyanionic polysaccharide strands of heparin bind more probe molecules per sugar unit when the charge of the latter is low, and stability of the probe-heparin complex increases with increasing probe charge. ${ }^{27}$ The red-fluorescent perylenediimide probe allowed sensitive quantification of heparin and LMWH in plasma and serum samples with minimal interference from matrix components. ${ }^{28}$ These are some of the new methods to determine heparins and were presented at the symposia at Villa Vigoni.

\section{Treatment of Venous Thromboembolism with Glycosaminoglycans: Special Indications}

Venous thromboembolism (VTE) is one of the relevant social burdens occurring as a complication of various underlying diseases leading to the postthrombotic syndrome if occurring in the extremities ${ }^{29}$ or to death in case of pulmonary embolism. ${ }^{30}$ The treatment of thromboembolic diseases is one of the main therapeutic areas in medicine, because up to $50 \%$ of patients with acute deep vein thrombosis will die without anticoagulant treatment within a few days. Reports on the prevention of postoperative VTE date back to the end of the 1930s. Crafoord reported the first prophylactic uses of repeated intravenous injections of heparin in postoperative medicine and in perinatal gynecology. ${ }^{31}$ Bauer, as reported by McLean, ${ }^{32}$ described the efficacy of heparin injection by reducing the incidence of mortality from 1 to $4 \%$ in 16,495 patients between 1939 and 1945 compared with 18\% without heparin observed between 1929 and 1938 at the Mariestad hospital in Sweden. In these series, a reduction of fatal pulmonary embolism was found from 47 of approximately 25,000 cases to 3 of approximately 16,000 cases. $^{32}$

According to more recent results, heparin reduced the mortality rate to less than $5 \%$. Unfractionated heparin (UFH) became the drug of choice in the mid-20th century for this indication. Because of the necessity of laboratory monitoring by the activated partial thromboplastin time, which had to be prolonged two to three times of the normal value LMWH have been developed.

Anticoagulation with heparin, LMWH, or fondaparinux is required to prevent VTE or to treat the acute event. ${ }^{33}$ The first report of the efficacy of heparin for postoperative prevention of VTE and for reduction of mortality were reported in the 1930s. LMWH showed an improved efficacy and reduced bleeding complications for prevention of VTE in postoperative care compared with UFH, ${ }^{34}$ in the prevention of myocardial infarction during an acute coronary syndrome, and for the treatment of acute VTE ${ }^{35}$ compared with UFH. In the initial treatment of VTE using LMWH once or twice daily subcutaneously, there did not appear any significant differences on the outcomes of recurrent VTE, major bleeding, or mortality. ${ }^{36}$ VTE is one of the major complications in patients suffering from cancer. A meta-analysis showed a reduction of VTE by $45 \%$ in cancer patients under treatment with LMWH and as a downside increased rates of bleeding complications ( $30 \%)$ compared with placebo. However, mortality rates were not reduced by LMWH compared with no anticoagulant treatment. ${ }^{37}$ Recently, effective anticoagulant treatments of thrombotic occlusion at unusual sites of retinal and jugular veins, right heart thrombosis including thrombosis of coronary sinus and thrombosis of the azygos system, umbilical, renal, ovarian, spermatic, and iliac veins were reported. ${ }^{38}$

\section{Modifications and Synthesis of Glycosaminoglycans}

Glycosaminoglycans have been modified because of the lack of oral absorption, adverse effects, and for investigations of structure function relationships with protein-binding. Some of the modifications took advantage of the end standing anhydromannose group of LMW heparins by synthetizing lipophilic residues ${ }^{39}$ with intact anticoagulant activity and prolonged elimination half-life after administration into rats. ${ }^{40}$ Despite the demonstration of the feasibility of this approach for improvement of the oral or transdermal administration, this concept of lipophilic modification of heparins did not reach a clinical stage of development. Fluorescence isothiocyanate labeled LMWH showed unaltered anticoagulant actions in vitro and after intravenous injection into rats. ${ }^{41}$ Binding to leukocytes may influence the pharmacology of LWMH also. This was demonstrated by the binding of this fluorescent labeled LMWH to granulocytes, monocytes, and lymphocytes in vitro and ex vivo after administration to rats and by a similar elimination half-life from these cells as compared with plasma. ${ }^{42}$

Seeberger and coworkers reported on the synthesis of different glycan structures, which were printed on 
microscope glass slides. The binding profiles on arrays of synthetic glycans and heparins identified adeno-associated viruses (AAV) serotype-specific capsid-carbohydrate interactions, allowing AAV capsid differentiation by glycan/heparinbinding patterns. ${ }^{43}$

\section{Nonanticoagulant Actions of Glycosaminoglycans}

The nonanticoagulant action of glycosaminoglycans plays a major role in their biological activity. A series of symposia at Villa Vigoni began in 1991 and covering many of these topics. The participants of the 21st symposium published several articles within the last year on the analysis, synthesis, and action of glycosaminoglycans.

Reduction of heparin in the presence of periodate oxidation/borohydride led to glycol-split heparins lacking anticoagulant and antithrombotic effects to be used for anticancer and anti-inflammation therapies. ${ }^{44}$ The nonanticoagulant action of glycol-split heparin inhibited hepcidin expression in hepatic HepG2 cells and primary hepatocytes and in mice suppressing liver hepcidin expression, reducing spleen iron, and reducing serum hepcidin levels. ${ }^{45}$ This was reproduced in an inflammation model with pretreatment with lipopolysaccharides followed by 1 -week treatments with glycol-split heparin. In a model of inflammatory anemia, glycol-split heparin increased iron mobilization and reduced anemia. These data indicate that high levels of hepcidin in anemia and autoimmune intestinal bowel diseases may be treated with these nonanticoagulant heparins. ${ }^{45}$

A modified sulfated nonanticoagulant heparin devoid of antithrombin (AT) binding and devoid of inhibition of systemic AT-dependent coagulation factors, and the LMWH tinzaparin both potently reduced adhesion and invasion of pancreatic cancer cells to the endothelial layer of umbilical cord vein in a dose-dependent manner. The sulfated heparin inhibited P-selectin mediated tumor cell adhesion, and inhibited cell adhesion and invasion similar to tinzaparin, indicating that systemic anticoagulation is not a necessary component for heparin attenuation of cancer cell adhesion, invasion, and metastasis. ${ }^{46}$ The integrin very late antigen (VLA-4) reduced metastasis production in a selectin-dependent manner. Nonanticoagulant heparin derivatives, enoxaparin, and tinzaparin effectively blocked VLA-4 cell binding, dominantly via the integrin's $\alpha$-chain. Desulfation at 2-Oposition, $\mathrm{N}$-acetylation, or a size smaller than tetradecasaccharide reduced VLA-4 inhibition. A derivative with 50\% 6-Odesulfation was more effective than tinzaparin. This can distinguish anticoagulant and antiadhesive functions of heparin for antimetastatic therapies without the risk of bleeding complications. ${ }^{47}$

In another tumor model, a murine mastocytoma cell line, which produce a highly sulfated heparin-like polysaccharide that lacks anticoagulant activity, transfection with a retroviral vector containing heparan sulfate 3-0-sulfotransferase-1 restored anticoagulant activity. These cell lines expressed $\mathrm{N}-$ acetylglucosamine $\mathrm{N}$-deacetylase/N-sulfotransferase-1, uronosyl 2-O-sulfotransferase, and glucosaminyl 6-O-sulfotrans- ferase-1, which was sufficient to make the highly sulfated heparin. Overexpression of this enzyme in mastocytoma cell lines resulted in a change in the composition of heparan sulfate/heparin and chondroitin sulfate/dermatan sulfate glycosaminoglycans. This model should provide a better understanding and a better control of the biosynthesis of heparin with different structures and activities. ${ }^{48} \mathrm{~A}$ series of polysulfated penta- and tetrasaccharide glycosides were synthesized as heparan sulfate mimetics and evaluated for their ability to inhibit angiogenesis. The compounds bound tightly to fibroblast growth factors (FGF-1, FGF-2, and vascular endothelial growth factor [VEGF]) and strongly inhibited heparanase activity. The compounds also showed good antitumor activity in vivo in a mouse melanoma (solid tumor) model. ${ }^{49}$ Extracellular vesicle-mediated intercellular transfer of signaling proteins and nucleic acids are involved in cancer development. Heparan sulfate proteoglycans function as internalizing receptors of exosomes, which are colocalized with cell-surface localized syndecan and glypican type. Exosome uptake was specifically inhibited by free heparan sulfate chains, by using several cell mutants, providing genetic evidence of a receptor function of exosome uptake, which was dependent on intact heparan sulfate, specifically on the 2-O and N-sulfation groups. Intact and not genetically modified heparan sulfate proteoglycans are key receptors for functional activity of exosomes in tumor growth. ${ }^{50}$

Heparanase is an endo- $\beta$-D-glucuronidase that cleaves heparan sulfate side chains of heparan sulfate proteoglycans on cell surfaces and the extracellular matrices in tumor metastasis and angiogenesis. Heparanase exerts also enzymatic-independent functions by upregulating VEGF-A, VEGF$\mathrm{C}$, and activation of intracellular signaling of cell survival and proliferation. Heparanase may also affect the hemostatic system in a nonenzymatic manner by expression of the blood coagulation initiator-TF and interaction with the TF pathway inhibitor (TFPI) on the cell surface membrane of endothelial and tumor cells. This leads to dissociation of TFPI and resulting in increased cell surface coagulation activity. It was shown that heparanase-enhanced TF activity resulted in factor Xa production. Inhibition of heparanase may be a good target for cancer therapy. ${ }^{51}$

The established role of heparanase in cancer and its newly discovered roles in diabetes, inflammation, and vascular diseases have elevated the importance of developing clinically effective antiheparanase therapies. Accomplishing this goal will require not only a deeper understanding of the heparanase mechanism of action in disease, but also resolution of heparanase structure and substrate specificity. ${ }^{52}$

Heparan sulfate was identified as a natural regulator of the cleavage of the amyloid precursor protein by $\beta$-secretase. During cleavage of the amyloid precursor protein, amyloid $\beta$-peptide is formed which is responsible for the formation of amyloid plaques present in the brains of Alzheimer patients. The deposition of insoluble accumulations of the amyloid $\beta$-peptide in the brain is critical in the development of Alzheimer disease. Heparan sulfate and nonanticoagulant heparins effectively inhibited $\beta$-secretase and progression of the disease. New insights into structure-activity 
relationships for optimal $\beta$-secretase inhibition were provided using a library of 12 - to 16 -mer oligosaccharides of heparan sulfate to improve treatment of Alzheimer disease. ${ }^{53}$

\section{Perspectives}

The analyses of the interactions of glycosaminoglycans with coagulation proteases as well as with nonanticoagulant proteins are currently improved by very specialized analytical methods. The synthesis of oligosaccharides of original and modified heparin-like products improves the understanding of specific interactions with proteins. The ultimate goal of these investigations is the development of defined glycosaminoglycans for treatment of nonthrombotic diseases.

\section{Conflict of Interest}

The authors do not have any conflicts of interest to declare.

\section{References}

1 Schmidt-Mülheim A. Beiträge zur Kenntnis des Peptons und seiner physiologischen Bedeutung. Vereinigung Wissenschaftlicher Verlage: Germany; 1880:33-56

2 McLean J. The thromboplastic action of cephalin. Am J Physiol 1916;41:250-257

3 Howell WH, Holt E. Two new factors in blood coagulation - heparin and prothrombin. Am J Physiol 1918;47:328-341

4 Howell $\mathrm{WH}$, McDonald $\mathrm{CH}$. Note on the effect of repeated intravascular injections of heparin. John Hopkins Hospital 1930; 46:365-371

5 Beller FK, Mammen E. Studies on a third plasma factor in thromboplastin formation [in German]. Klin Wochenschr 1955;33(7-8): 155-156

6 Nieth H, Mammen E, Gross R. Heparinization of blood in extracorporeal hemodialysis [in German]. Klin Wochenschr 1958;36(8): 372-376

7 Troy S, Fruncillo R, Ozawa T, Mammen E, Holloway S, Chiang S. The dose proportionality of the pharmacokinetics of ardeparin, a low molecular weight heparin, in healthy volunteers. J Clin Pharmacol 1995;35(12):1194-1199

8 Mammen EF. Why low molecular weight heparin? Semin Thromb Hemost 1990;16(Suppl):1-4

9 Harenberg J, Casu B. Glycosaminoglycans I. Semin Thromb Hemost 1994;20:133-222

10 Harenberg J, Casu B. Glycosaminoglycans: anticoagulant and nonanticoagulant actions. Semin Thromb Hemost 2002;28:323-402

11 Harenberg J, Casu B (Guest Editors). New anticoagulants. [Complete Issue]. Semin Thromb Hemost 2007;33(5):449-562

12 Beeler D, Rosenberg R, Jordan R. Fractionation of low molecular weight heparin species and their interaction with antithrombin. J Biol Chem 1979;254(8):2902-2913

13 Petitou M, Duchaussoy P, Herbert JM, et al. The synthetic pentasaccharide fondaparinux: first in the class of antithrombotic agents that selectively inhibit coagulation factor Xa. Semin Thromb Hemost 2002;28(4):393-402

14 Viskov C, Elli S, Urso E, et al. Heparin dodecasaccharide containing two antithrombin-binding pentasaccharides: structural features and biological properties. J Biol Chem 2013;288(36):25895-25907

15 Malsch R, Harenberg J. High-performance size exclusion chromatography and polyacrylamide gel electrophoresis for characterization of unfractionated and low molecular mass glycosaminoglycans. Semin Thromb Hemost 1994;20(2):135-143
16 Ucakturk E, Cai C, Li L, Li G, Zhang F, Linhardt RJ. Capillary electrophoresis for total glycosaminoglycan analysis. Anal Bioanal Chem 2014;406(19):4617-4626

17 Dinges MM, Solakyildirim K, Larive CK. Affinity capillary electrophoresis for the determination of binding affinities for low molecular weight heparins and antithrombin-III. Electrophoresis 2014; 35(10):1469-1477

18 Rudd TR, Skidmore MA, Guimond SE, et al. Glycosaminoglycan origin and structure revealed by multivariate analysis of NMR and CD spectra. Glycobiology 2009;19(1):52-67

19 Sturiale L, Naggi A, Torri G. MALDI mass spectrometry as a tool for characterizing glycosaminoglycan oligosaccharides and their interaction with proteins. Semin Thromb Hemost 2001;27(5): 465-472

20 Bultel L, Landoni M, Grand E, Couto AS, Kovensky J. UV-MALDI-TOF mass spectrometry analysis of heparin oligosaccharides obtained by nitrous acid controlled degradation and high performance anion exchange chromatography. J Am Soc Mass Spectrom 2010;21(1):178-190

21 Ratnoff OD, Menzie C. A new method for the determination of fibrinogen in small samples of plasma. J Lab Clin Med 1951;37(2): 316-320

22 O'Connor SD, Taylor AJ, Williams EC, Winter TC. Coagulation concepts update. AJR Am J Roentgenol 2009;193(6):1656-1664

23 Favaloro EJ, Lippi G, Koutts J. Laboratory testing of anticoagulants: the present and the future. Pathology 2011;43(7):682-692

24 Harenberg J, Giese C, Hagedorn A, Traeger I, Fenyvesi T. Determination of antithrombin-dependent factor Xa inhibitors by prothrombin-induced clotting time. Semin Thromb Hemost 2007; 33(5):503-507

25 Alban S. Adverse effects of heparin. Handbook Exp Pharmacol 2012;(207):211-263

26 Szelke H, Harenberg J, Krämer R. Detection and neutralisation of heparin by a fluorescent ruthenium compound. Thromb Haemost 2009;102(5):859-864

27 Szelke H, Schübel S, Harenberg J, Krämer R. Interaction of heparin with cationic molecular probes: probe charge is a major determinant of binding stoichiometry and affinity. Bioorg Med Chem Lett 2010;20(4):1445-1447

28 Szelke H, Schübel S, Harenberg J, Krämer R. A fluorescent probe for the quantification of heparin in clinical samples with minimal matrix interference. Chem Commun (Camb) 2010;46(10):1667-1669

29 Kahn SR, Ginsberg JS. Relationship between deep venous thrombosis and the postthrombotic syndrome. Arch Intern Med 2004; 164(1):17-26

30 Tagalakis V, Patenaude V, Kahn SR, Suissa S. Incidence of and mortality from venous thromboembolism in a real-world population: the Q-VTE Study Cohort. Am J Med 2013;126(9):e13-e21

31 Crafoord C. Heparin and post-operative thrombosis. Acta Chir Scand 1939;82:319-335

32 McLean J. The discovery of heparin. Circulation 1959;19(1):75-78

33 Wells PS, Forgie MA, Rodger MA. Treatment of venous thromboembolism. JAMA 2014;311(7):717-728

34 Kearon C, Akl EA, Comerota AJ, et al; American College of Chest Physicians. Antithrombotic therapy for VTE disease: antithrombotic therapy and prevention of thrombosis, 9th ed: american college of chest physicians evidence-based clinical Practice Guidelines. Chest 2012;141(2, Suppl):e419S-e494S

35 Kearon C. A conceptual framework for two phases of anticoagulant treatment of venous thromboembolism. J Thromb Haemost 2012; 10(4):507-511

36 Bhutia S, Wong PF. Once versus twice daily low molecular weight heparin for the initial treatment of venous thromboembolism. Cochrane Database Syst Rev 2013;7:CD003074

37 Che DH, Cao JY, Shang LH, Man YC, Yu Y. The efficacy and safety of low-molecular-weight heparin use for cancer treatment: a metaanalysis. Eur J Intern Med 2013;24(5):433-439 
38 Girolami A, Treleani M, Bonamigo E, Tasinato V, Girolami B. Venous thrombosis in rare or unusual sites: a diagnostic challenge. Semin Thromb Hemost 2014;40(1):81-87

39 Harenberg J. Development of new anticoagulants: present and future. Semin Thromb Hemost 2008;34(8):779-793

40 Malsch R, Harenberg J, Guerrini M, Torri G, Casu B, Heene DL. Semisynthesis and analysis of lipophilically modified unfractionated and low molecular mass heparins. Semin Thromb Hemost 1994;20(2):182-192

41 Piazolo L, Harenberg J, Malsch R, Heene DL. Binding of fluorescentlabeled low molecular mass heparin to latex microspheres and to rat and human leukocytes. Semin Thromb Hemost 1994;20(3):227-235

42 Harenberg J, Casu B, Guerrini M, et al. Low-molecular-weight heparin and dermatan sulfate end group-labeled with tyramine and fluorescein. Biochemical and biological characterization of the fluorescent-labeled heparin derivative. Semin Thromb Hemost 2002;28(4):343-354

43 Mietzsch M, Broecker F, Reinhardt A, Seeberger PH, Heilbronn R. Differential adeno-associated virus serotype-specific interaction patterns with synthetic heparins and other glycans. J Virol 2014; 88(5):2991-3003

44 Alekseeva A, Casu B, Torri G, Pierro S, Naggi A. Profiling glycol-split heparins by high-performance liquid chromatography/mass spectrometry analysis of their heparinase-generated oligosaccharides. Anal Biochem 2013;434(1):112-122

45 Poli M, Asperti M, Naggi A, et al. Glycol-split nonanticoagulant heparins are inhibitors of hepcidin expression in vitro and in vivo. Blood 2014;123(10):1564-1573
46 Sudha T, Phillips P, Kanaan C, Linhardt RJ, Borsig L, Mousa SA. Inhibitory effect of non-anticoagulant heparin (S-NACH) on pancreatic cancer cell adhesion and metastasis in human umbilical cord vessel segment and in mouse model. Clin Exp Metastasis 2012;29(5):431-439

47 Schlesinger M, Schmitz P, Zeisig R, et al. The inhibition of the integrin VLA-4 in MV3 melanoma cell binding by non-anticoagulant heparin derivatives. Thromb Res 2012;129(5):603-610

48 Gasimli L, Glass CA, Datta P, et al. Bioengineering murine mastocytoma cells to produce anticoagulant heparin. Glycobiology 2014;24(3):272-280

49 Johnstone KD, Karoli T, Liu L, et al. Synthesis and biological evaluation of polysulfated oligosaccharide glycosides as inhibitors of angiogenesis and tumor growth. J Med Chem 2010;53(4): 1686-1699

50 Christianson HC, Svensson KJ, van Kuppevelt TH, Li JP, Belting M. Cancer cell exosomes depend on cell-surface heparan sulfate proteoglycans for their internalization and functional activity. Proc Natl Acad Sci USA 2013;110(43):17380-17385

51 Nadir Y, Brenner B. Heparanase multiple effects in cancer. Thromb Res 2014;133(Suppl 2):S90-S94

52 Vlodavsky I, Iozzo RV, Sanderson RD. Heparanase: multiple functions in inflammation, diabetes and atherosclerosis. Matrix Biol 2013;32(5):220-222

53 Schwörer R, Zubkova OV, Turnbull JE, Tyler PC. Synthesis of a targeted library of heparan sulfate hexa- to dodecasaccharides as inhibitors of $\beta$-secretase: potential therapeutics for Alzheimer's disease. Chemistry 2013;19(21):6817-6823 